\title{
NUIG: Multitasking Self-attention based approach to SigTyp 2020 Shared Task
}

\author{
Chinmay Choudhary \\ National University of Ireland, Galway \\ c. choudharyl@nuigalway.ie
}

\author{
Colm O'Riordan \\ National University of Ireland, Galway \\ colm.oriordanenuigalway. ie
}

\begin{abstract}
The paper describes the Multitasking Selfattention based approach to constrained subtask within Sigtyp 2020 Shared task. Our model is simple neural network based architecture inspired by Transformers (Vaswani et al., 2017) model. The model uses Multitasking to compute values of all WALS features for a given input language simultaneously.

Results show that our approach performs at par with the baseline approaches, even though our proposed approach requires only phylogenetic and geographical attributes namely Longitude, Latitude, Genus-index, Family-index and Country-index and do not use any of the known WALS features of the respective input language, to compute its missing WALS features.
\end{abstract}

\section{Introduction}

In this paper we describe our Multitasking Selfattention based approach to Sigtyp 2020 Shared task (Constrained Sub-task) (Bjerva et al., 2020) which involves prediction of values of features from WALS Typology database for various lowresourced languages.

Linguistic typology is the classification of human languages according to their syntactic, phonological and semantic features. WALS (Haspelmath, 2009) is the most popular and comprehensive database which provides list of typological features and their possible values, as well as the respective feature-values for most of the world's languages. However all the popular typological databases (Haspelmath, 2009; Collins and Kayne, 2009; Maddieson et al., 2013; Hartmann and Bradley Taylor, 2013; Bickel et al., 2017; Michaelis and Magnus Huber, 2013) (including WALS) suffer from a major shortcoming of limited coverage. In fact, values of many important typological features for most languages (specially less documented ones) are missing in these databases. This sparked a line of research on automatic acquisition of such missing typology knowledge (Malaviya et al., 2017; Coke et al., 2016; Daumé III, 2009; Daumé III and Campbell, 2009; Littell et al., 2017; Bjerva et al., 2019).

Our proposed model is a neural network architecture which takes in as input, the phylogenetic and geographical attributes of a language. The model subsequently predicts values of all its typology features simultaneously using Multitask learning setup (Ruder, 2017).

\section{Model}

Figure 1 depicts the architecture of our proposed model that computes values of all WALS typology features for a given language simultaneously. As evident in Figure 1, our proposed model architecture comprises of three components namely Input Network Component, Self-attention Network Component and Multitasking Output Networks Component described as section 2.1, 2.2 and 2.3 respectively.

\subsection{Input Network Component}

The input component is a simple two layered feedforward neural network. The input of the network is a 5-dimensional vector $x$ comprising of values of five key attributes of any language, namely Longitude, Latitude, Genus-index, Family-index and Country-index as these are the attributes provided by train and test datasets (for all languages within the datasets) for Sigtyp 2020 Shared Task. We computed Genus-index, Family-index and Countryindex from genus, family and countryCode attributes provided within dataset using respective name-index dictionaries.

This two layered feed forward network computes output vector $o \in R^{T * d}$ where $\mathrm{T}$ is the total number of WALS typology features to be predicted by 


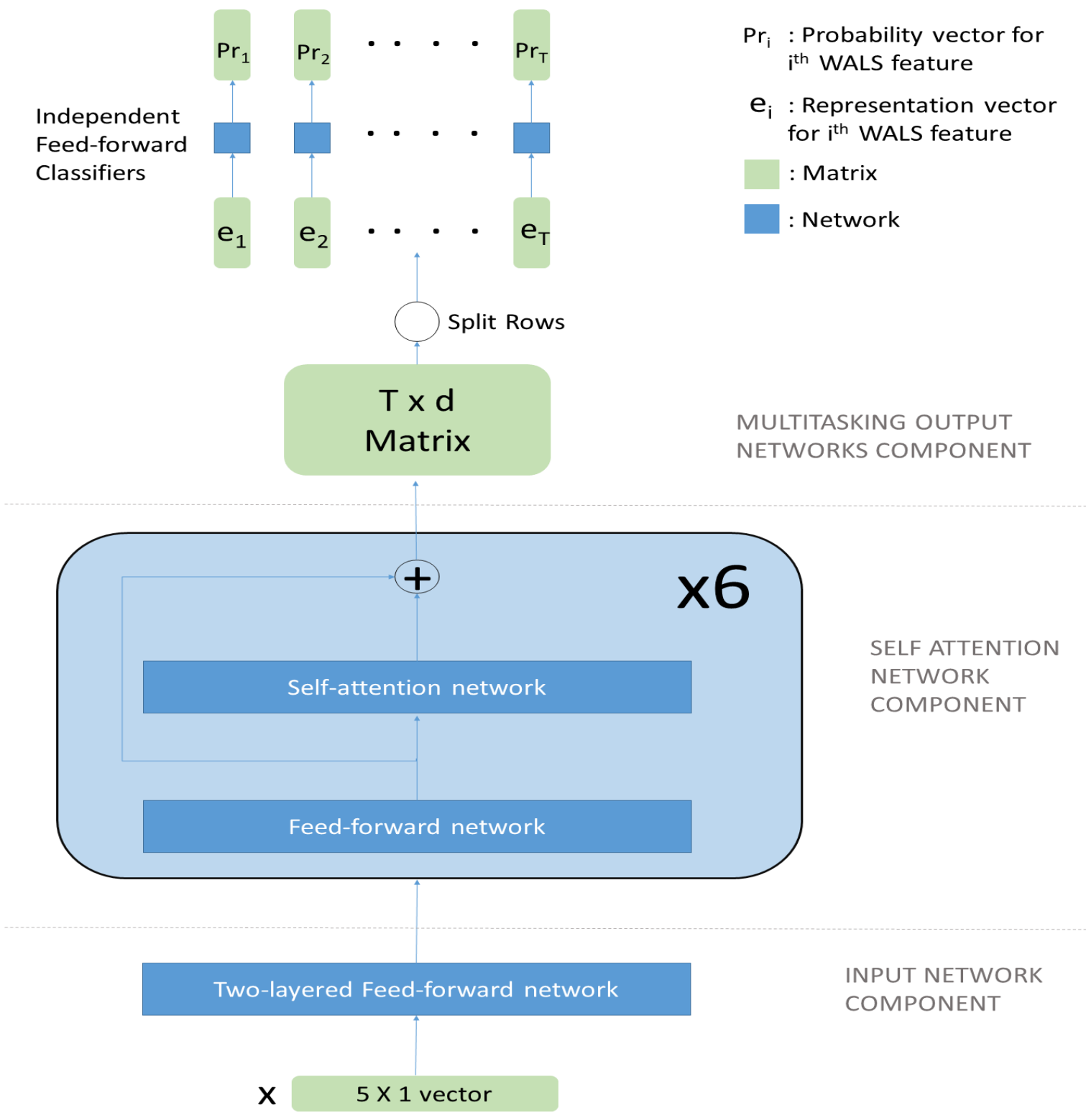

Figure 1: Architecture of proposed model

applying equations 1 and 2 .

$$
\begin{gathered}
\hat{o}=\tanh \left(A_{1} * x+a_{1}\right) \\
o=\tanh \left(A_{2}{ }^{T} * \hat{o}+a_{2}\right)
\end{gathered}
$$

Here $A_{1} \in R^{d * 5}, A_{2} \in R^{T * 1}$ are weights and $a_{1} \in R^{d}$ and $a_{2} \in R^{T * d}$ are biases.

\subsection{Self-attention Network Component}

The architecture of this component is inspired by Transformers (Vaswani et al., 2017) model. The model architecture comprises a stack of $N=6$ identical layers. Each layer has two sub-layers. The first is a multi-head self-attention mechanism, and the second is a simple fully connected feed-forward network. Hence input to layer $i$ is always the output from layer $i-1$. Input to the first layer is the output of the previous Input Network Component.

For $i^{\text {th }}$ layer within architecture, its Feed-forward and self-attention sub-layers are given by equations 3 and 4.

$$
\begin{gathered}
h_{i}=\tanh \left(W_{i} * y_{i-1}+b_{i}\right) \\
k_{i}=\operatorname{attention}\left(h_{i}, h_{i}\right)
\end{gathered}
$$

Here $h_{i} \in R^{d}$ and $k_{i} \in R^{d}$ are outputs of feed-forward and self-attention layers respectively. We used same attention mechanism as used by (Vaswani et al., 2017). Final output of $i^{\text {th }}$ layer 


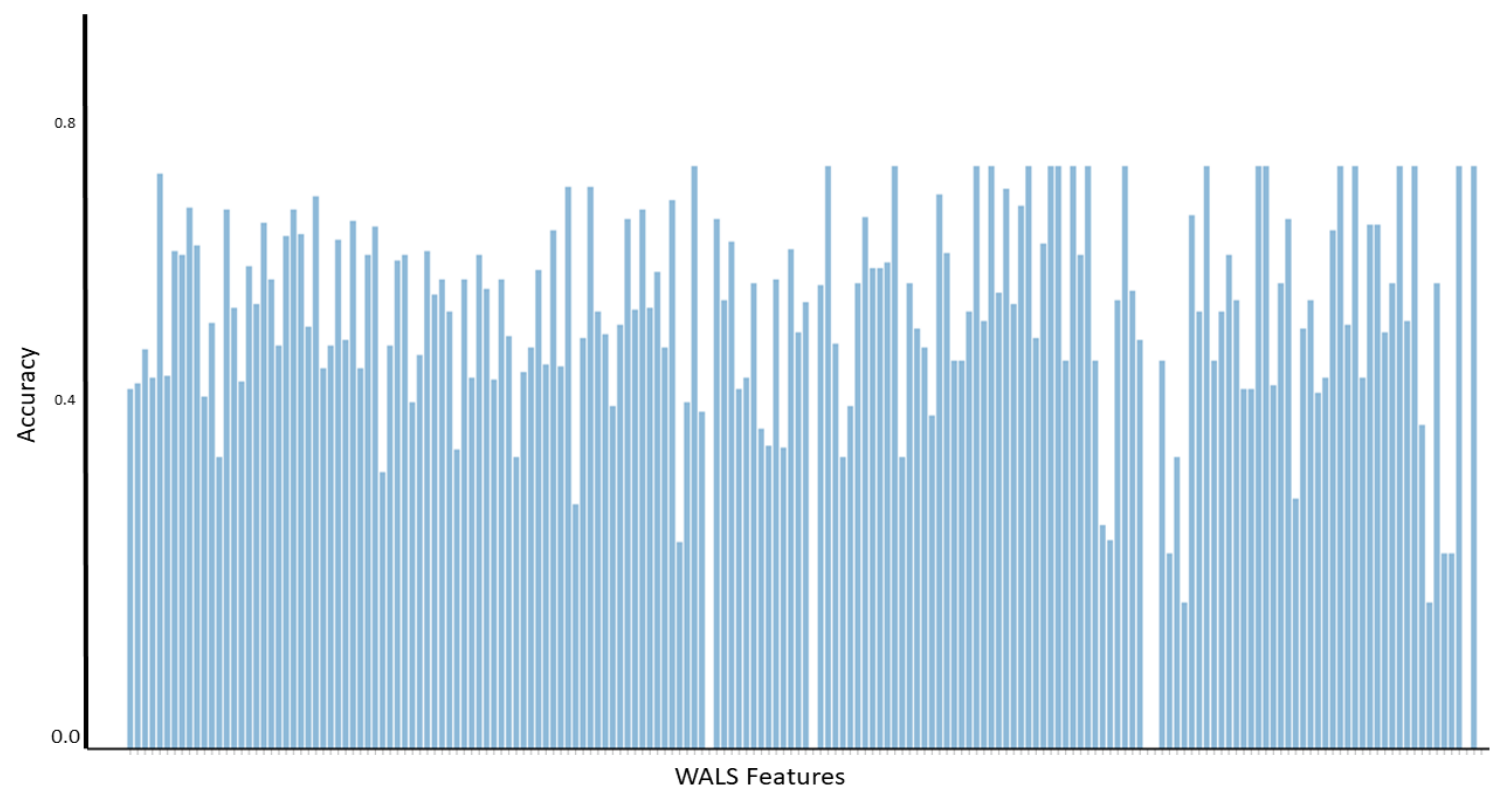

Figure 2: Plot depicting trend in accuracy values achieved on all WALS features

$y_{i}$ is computed by adding $h_{i}$ and $k_{i}$ (equation 3 ).

$$
y_{i}=h_{i}+k_{i}
$$

Input to the first layer $y_{0}$ is the output from previous Input Network Component. Output of Selfattention Network Component is the output of final layer $y_{N}$.

It is been observed that there is a correlation between various WALS typology features. Thus, to predict the missing value of a particular typology feature for a specific languages, knowledge about other typology features for that languages would be useful. Such knowledge is ensured by the selfattention layers.

\subsection{Multitasking Output Networks Component}

Multitasking Output Networks Component comprises of $T$ independent feed-forward neuralnetwork classifiers. The component splits the output of previous Self-attention Network Component i.e $y_{6} \in R^{T * d}$ into $\mathrm{T}$ d-dimensional vectors $e_{1}, e_{2}, \ldots e_{T}$. each corresponds to one of the $\mathrm{T}$ typology features to be predicted.

Value of the $j^{t h}$ typology feature is computed by applying equation 6 .

$$
P r_{j}=\operatorname{Softmax}\left(W_{j} * e_{j}+c_{j}\right)
$$

Here $1<=j<=T, \operatorname{Pr}_{j}$ provides probability of each of the possible values for $j^{\text {th }}$ typology feature being the true-value. Dimensions of weights and

\begin{tabular}{|c|c|}
\hline Hyper-parameter & Value \\
\hline $\mathrm{d}$ & 548 \\
\hline drop_out probability & 0.1 \\
\hline learning_rate & 0.1 \\
\hline reduce lr on plateau & Yes \\
\hline reduce factor & 0.001 \\
\hline batch-size & 20 \\
\hline steps-per-epoch & 50 \\
\hline epochs & 200 \\
\hline Number of features $(\mathrm{T})$ & 185 \\
\hline
\end{tabular}

Table 1: Hyper-parameters

biases are unique for each classifier as number of possible values for each of the typology features is unique.

\section{Training}

The parameters of model described in section 2 are trained by optimizing the loss function given by equation 7 .

$$
\text { Loss }=\Sigma_{t=1}^{T} C E\left(P r_{t}, O H_{t}\right)
$$

Here $O H_{t}$ is the one-hot encoding of true-value for $t^{t h}$ typology feature. CE is the Cross-entropy loss. Table 1 lists the hyper-parameters used during training. These are computed by minimizing the loss over Validation set. 


\begin{tabular}{|c|c|}
\hline Model & Accuracy \\
\hline frequency-baseline_constrained & 0.514 \\
\hline knn-imputation-baseline_constrained & 0.508 \\
\hline NUIG_constrained & 0.487 \\
\hline
\end{tabular}

Table 2: Overall Accuracy of baseline and proposed models

\section{Results}

Table 2 compared the accuracy achieved by our proposed model with two baselines provided namely frequency-baseline-constrained and knnimputation-baseline-constrained.

It is evident from table that our model performs at par with baselines, even though it utilizes only five attributes of the input language, namely Longitude, Latitude, Genus-index, Family-index and Country-index (model doesn't utilize any known WALS feature values, provided within test dataset for various languages).

Figure 2 is bar-plot that depicts the trend in accuracy achieved by our model on various WALS features. Precise accuracy score achieved by our model on all 185 WALS typology features is provided in Appendix.

\section{Conclusion}

In this paper we described our Multitasking Selfattention based approach to Sigtyp 2020 Shared task, Constrained Sub-task. Our model is simple neural network based approach which computes values of all WALS features for a given input language simultaneously in Multitasking settings. The architecture of our network is inspired by Transformers (Vaswani et al., 2017).

Results show that our approach performs at par with the baseline approaches, even though our approach uses only five linguistic and geographical attributes namely Longitude, Latitude, Genus-index, Family-index and Country-index and do not use any of the known WALS features of the respective input language, to compute its missing WALS features.

\section{References}

Balthasar Bickel, Johanna Nichols, Taras Zakharko, Alena Witzlack-Makarevich, Fernando Hildebrandt, Kristine, and John B Lowe. 2017. The autotyp typological databases. Version 0.1. 0. Online: https://github.com/autotyp/ autotyp-data/tree/0.1.0.
Johannes Bjerva, Yova Kementchedjhieva, Ryan Cotterell, and Isabelle Augenstein. 2019. A probabilistic generative model of linguistic typology. arXiv preprint arXiv:1903.10950.

Johannes Bjerva, Elizabeth Salesky, Sabrina Mielke, Aditi Chaudhary, Giuseppe G. A. Celano, Edoardo M. Ponti, Ekaterina Vylomova, Ryan Cotterell, and Isabelle Augenstein. 2020. SIGTYP 2020 Shared Task: Prediction of Typological Features. In Proceedings of the Second Workshop on Computational Research in Linguistic Typology. Association for Computational Linguistics.

Reed Coke, Ben King, and Dragomir Radev. 2016. Classifying syntactic regularities for hundreds of languages. arXiv preprint arXiv:1603.08016.

Chris Collins and Richard Kayne. 2009. Syntactic structures of the world's languages. http://sswl. railsplayground.net/..

Hal Daumé III. 2009. Non-parametric bayesian areal linguistics. arXiv preprint arXiv:0906.5114.

Hal Daumé III and Lyle Campbell. 2009. A bayesian model for discovering typological implications. arXiv preprint arXiv:0907.0785.

Martin Haspelmath Hartmann, Iren and editors Bradley Taylor. 2013. Valency Patterns Leipzig.

Martin Haspelmath. 2009. The typological database of the World Atlas of Language Structures. Berlin: Walter de Gruyter.

Patrick Littell, David R Mortensen, Ke Lin, Katherine Kairis, Carlisle Turner, and Lori Levin. 2017. Uriel and lang2vec: Representing languages as typological, geographical, and phylogenetic vectors. In Proceedings of the 15th Conference of the European Chapter of the Association for Computational Linguistics: Volume 2, Short Papers, pages 8-14.

Ian Maddieson, Sébastien Flavier, Egidio Marsico, Christophe Coupé, and François Pellegrino. 2013. Lapsyd: lyon-albuquerque phonological systems database. In INTERSPEECH, pages 3022-3026.

Chaitanya Malaviya, Graham Neubig, and Patrick Littell. 2017. Learning language representations for typology prediction. arXiv preprint arXiv:1707.09569.

Philippe Maurer Martin Haspelmath Michaelis, Susanne Maria and editors Magnus Huber. 2013. Atlas of Pidgin and Creole Language Structures Online.

Sebastian Ruder. 2017. An overview of multitask learning in deep neural networks. corr abs/1706.05098. arXiv preprint arXiv:1706.05098.

Ashish Vaswani, Noam Shazeer, Niki Parmar, Jakob Uszkoreit, Llion Jones, Aidan N Gomez, Łukasz Kaiser, and Illia Polosukhin. 2017. Attention is all you need. In Advances in neural information processing systems, pages 5998-6008. 


\section{A Appendix 1}

Table 3: Feature-wise accuracy.

\begin{tabular}{|c|c|}
\hline \multicolumn{2}{|l|}{ Begin of Table } \\
\hline WALS Feature & Accuracy \\
\hline Order_of_Person_Markers_on_the_Verb & 0.4307692307692308 \\
\hline Order_of_Subject,_Object_and_Verb & 0.43855421686746987 \\
\hline Order_of_Adposition_and_Noun_Phrase & 0.4805970149253731 \\
\hline Position_of_Case_Affixes & 0.4445945945945946 \\
\hline Minor_morphological_means_of_signaling_negation & 0.6905405405405406 \\
\hline Position_of_Tense-Aspect_Affixes & 0.5986842105263158 \\
\hline Order_of_Degree_Word_and_Adjective & 0.5923076923076923 \\
\hline Postnominal_relative_clauses & 0.65 \\
\hline Postverbal_Negative_Morphemes & 0.6054054054054054 \\
\hline Person_Marking_on_Adpositions & 0.5115384615384615 \\
\hline Weight_Factors_in_Weight-Sensitive_Stress_Systems & 0.35 \\
\hline Presence_of_Uncommon_Consonants & 0.6487804878048781 \\
\hline Preverbal_Negative_Morphemes & 0.5297297297297298 \\
\hline Negative_Morphemes & 0.44 \\
\hline Absence_of_Common_Consonants & 0.5804878048780487 \\
\hline Polar_Questions & 0.5345454545454545 \\
\hline Glottalized_Consonants & 0.6317073170731707 \\
\hline Voicing_in_Plosives_and_Fricatives & 0.5634146341463415 \\
\hline Position_of_Negative_Word_With_Respect_to_Subject,_Object,_and_Verb & 0.4846153846153846 \\
\hline Passive_Constructions & 0.616 \\
\hline Front_Rounded_Vowels & 0.6487804878048781 \\
\hline Gender_Distinctions_in_Independent_Personal_Pronouns & 0.6192307692307693 \\
\hline Rhythm_Types & 0.506896551724138 \\
\hline Tone & 0.6631578947368421 \\
\hline Position_of_Polar_Question_Particles & 0.45769230769230773 \\
\hline Order_of_Numeral_and_Noun & 0.4830985915492958 \\
\hline Pronominal_and_Adnominal_Demonstratives & 0.6125 \\
\hline Fixed_Stress_Locations & 0.49 \\
\hline Finger_and_Hand & 0.6348837209302325 \\
\hline Verbal_Person_Marking & 0.45769230769230773 \\
\hline Third_Person_Zero_of_Verbal_Person_Marking & 0.5923076923076923 \\
\hline Order_of_Subject_and_Verb & 0.6263157894736842 \\
\hline Weight-Sensitive_Stress & 0.3325 \\
\hline Order_of_Object_and_Verb & 0.48404255319148937 \\
\hline Order_of_Relative_Clause_and_Noun & 0.586046511627907 \\
\hline Alignment_of_Verbal_Person_Marking & 0.5923076923076923 \\
\hline Position_of_Pronominal_Possessive_Affixes & 0.4148148148148148 \\
\hline Voicing_and_Gaps_in_Plosive_Systems & 0.5975609756097561 \\
\hline Consonant-Vowel_Ratio & 0.5463414634146342 \\
\hline Expression_of_Pronominal_Subjects & 0.5630434782608695 \\
\hline Intensifiers_and_Reflexive_Pronouns & 0.525 \\
\hline Consonant_Inventories & 0.35853658536585364 \\
\hline Vowel_Quality_Inventories & 0.5634146341463415 \\
\hline Syllable_Structure & 0.44545454545454544 \\
\hline
\end{tabular}




\begin{tabular}{|c|c|}
\hline \multicolumn{2}{|l|}{ Continuation of Table 3} \\
\hline WALS Feature & Accuracy \\
\hline Order_of_Genitive_and_Noun & 0.5936708860759494 \\
\hline Order_of_Adjective_and_Noun & 0.5530864197530864 \\
\hline Third_Person_Pronouns_and_Demonstratives & 0.4421052631578947 \\
\hline Lateral_Consonants & 0.5634146341463415 \\
\hline Prefixing_vs._Suffixing_in_Inflectional_Morphology & 0.49482758620689654 \\
\hline The_Position_of_Negative_Morphemes_in_Object-Initial_Languages & 0.35 \\
\hline Distance_Contrasts_in_Demonstratives & 0.4529411764705883 \\
\hline Order_of_Negative_Morpheme_and_Verb & 0.4824324324324324 \\
\hline Hand_and_Arm & 0.574 \\
\hline Uvular_Consonants & 0.4609756097560976 \\
\hline Position_of_Interrogative_Phrases_in_Content_Questions & 0.6234375000000001 \\
\hline SONegV_Order & 0.45862068965517244 \\
\hline NegSOV_Order & 0.6758620689655173 \\
\hline The_Position_of_Negative_Morphemes_in_SOV_Languages & 0.2930232558139535 \\
\hline The_Associative_Plural & 0.49411764705882355 \\
\hline SNegOV_Order & 0.6758620689655173 \\
\hline Order_of_Adverbial_Subordinator_and_Clause & 0.525 \\
\hline The_Prohibitive & 0.49677419354838714 \\
\hline SOVNeg_Order & 0.4117647058823529 \\
\hline Coding_of_Nominal_Plurality & 0.5101694915254237 \\
\hline The_Morphological_Imperative & 0.6363636363636364 \\
\hline Order_of_Demonstrative_and_Noun & 0.5272727272727273 \\
\hline Comitatives_and_Instrumentals & 0.6481481481481481 \\
\hline Ditransitive_Constructions:_The_Verb_'Give' & 0.5303030303030303 \\
\hline 'Want'_Complement_Subjects & 0.5727272727272728 \\
\hline Order_of_Object,_Oblique,_and_Verb & 0.48125 \\
\hline Noun_Phrase_Conjunction & 0.6588235294117647 \\
\hline Predicative_Possession & 0.24705882352941178 \\
\hline Definite_Articles & 0.41621621621621624 \\
\hline Languages_with_two_Dominant_Orders_of_Subject,_Object,_and_Verb & 0.7 \\
\hline Zero_Copula_for_Predicate_Nominals & 0.40384615384615385 \\
\hline Languages_with_different_word_order_in_negative_clauses & 0.0 \\
\hline Tea & 0.6363636363636364 \\
\hline Nominal_and_Locational_Predication & 0.5384615384615385 \\
\hline Predicative_Adjectives & 0.4307692307692308 \\
\hline Nominal_and_Verbal_Conjunction & 0.44545454545454544 \\
\hline Cultural_Categories_of_Languages_with_Identity_of_'Finger'_and_'Hand' & 0.56 \\
\hline Inclusive/Exclusive_Forms_in_Pama-Nyungan & 0.385 \\
\hline Occurrence_of_Nominal_Plurality & 0.364 \\
\hline Indefinite_Pronouns & 0.564516129032258 \\
\hline Indefinite_Articles & 0.3612903225806452 \\
\hline The_Optative & 0.6 \\
\hline Ordinal_Numerals & 0.5 \\
\hline Semantic_Distinctions_of_Evidentiality & 0.5352941176470588 \\
\hline Multiple_Negative_Constructions_in_SOV_Languages & 0.0 \\
\hline Coding_of_Evidentiality & 0.5558823529411765 \\
\hline Politeness_Distinctions_in_Pronouns & 0.7 \\
\hline Systems_of_Gender_Assignment & 0.4869565217391304 \\
\hline
\end{tabular}




\begin{tabular}{|c|c|}
\hline \multicolumn{2}{|l|}{ Continuation of Table 3} \\
\hline WALS Feature & Accuracy \\
\hline Locus_of_Marking:_Whole-language_Typology & 0.35 \\
\hline Asymmetrical_Case-Marking & 0.4117647058823529 \\
\hline M_in_First_Person_Singular & 0.56 \\
\hline Sex-based_and_Non-sex-based_Gender_Systems & 0.6391304347826087 \\
\hline Number_of_Cases & 0.5764705882352941 \\
\hline Reduplication & 0.5764705882352941 \\
\hline Numeral_Classifiers & 0.58333333333333334 \\
\hline Number_of_Possessive_Nouns & 0.7 \\
\hline Applicative_Constructions & 0.35 \\
\hline M_in_Second_Person_Singular & 0.56 \\
\hline Locus_of_Marking_in_Possessive_Noun_Phrases & 0.5055555555555555 \\
\hline Possessive_Classification & 0.48125 \\
\hline Other_Roles_of_Applied_Objects & 0.4 \\
\hline M-T_Pronouns & 0.665 \\
\hline N-M_Pronouns & 0.595 \\
\hline Locus_of_Marking_in_the_Clause & 0.4666666666666667 \\
\hline Adjectives_without_Nouns & 0.4666666666666667 \\
\hline Antipassive_Constructions & 0.525 \\
\hline Zero_Marking_of_A_and_P_Arguments & 0.7 \\
\hline Productivity_of_the_Antipassive_Construction & 0.51333333333333333 \\
\hline Obligatory_Possessive_Inflection & 0.7 \\
\hline Number_of_Genders & 0.5478260869565218 \\
\hline Nonperiphrastic_Causative_Constructions & 0.6719999999999999 \\
\hline Plurality_in_Independent_Personal_Pronouns & 0.5333333333333333 \\
\hline Purpose_Clauses & 0.65333333333333333 \\
\hline Imperative-Hortative_Systems & 0.7 \\
\hline Vowel_Nasalization & 0.49411764705882355 \\
\hline Prenominal_relative_clauses & 0.6066666666666667 \\
\hline SVNegO_Order & 0.7 \\
\hline SVONeg_Order & 0.7 \\
\hline Number_of_Non-Derived_Basic_Colour_Categories & 0.4666666666666667 \\
\hline Red_and_Yellow & 0.7 \\
\hline NegSVO_Order & 0.5923076923076923 \\
\hline Green_and_Blue & 0.7 \\
\hline Number_of_Basic_Colour_Categories & 0.46666666666666667 \\
\hline SNegVO_Order & 0.2692307692307693 \\
\hline The_Position_of_Negative_Morphemes_in_SVO_Languages & 0.25 \\
\hline Negative_Indefinite_Pronouns_and_Predicate_Negation & 0.5384615384615385 \\
\hline Utterance_Complement_Clauses & 0.7 \\
\hline 'When'_Clauses & 0.55 \\
\hline The_Velar_Nasal & 0.49 \\
\hline Optional_Double_Negation_in_SOV_languages & 0.0 \\
\hline Optional_Double_Negation & 0.0 \\
\hline Para-Linguistic_Usages_of_Clicks & 0.4666666666666667 \\
\hline Distributive_Numerals & 0.23333333333333334 \\
\hline Verb-Initial_with_Preverbal_Negative & 0.35 \\
\hline The_Position_of_Negative_Morphemes_in_Verb-Initial_Languages & 0.175 \\
\hline Reason_Clauses & 0.6416666666666666 \\
\hline
\end{tabular}




\begin{tabular}{|c|c|}
\hline \multicolumn{2}{|l|}{ Continuation of Table 3} \\
\hline WALS Feature & Accuracy \\
\hline Verb-Initial_with_Clause-Final_Negative & 0.7 \\
\hline Alignment_of_Case_Marking_of_Pronouns & 0.46666666666666667 \\
\hline Alignment_of_Case_Marking_of_Full_Noun_Phrases & 0.525 \\
\hline Inclusive/Exclusive_Distinction_in_Verbal_Inflection & 0.5923076923076923 \\
\hline Syncretism_in_Verbal_Person/Number_Marking & 0.5384615384615385 \\
\hline Numeral_Bases & 0.4307692307692308 \\
\hline Case_Syncretism & 0.4307692307692308 \\
\hline Inclusive/Exclusive_Distinction_in_Independent_Pronouns & 0.7 \\
\hline Relativization_on_Subjects & 0.7 \\
\hline Action_Nominal_Constructions & 0.4375 \\
\hline Periphrastic_Causative_Constructions & 0.56 \\
\hline Situational_Possibility & 0.6363636363636364 \\
\hline Relativization_on_Obliques & 0.3 \\
\hline Symmetric_and_Asymmetric_Standard_Negation & 0.5055555555555555 \\
\hline Epistemic_Possibility & 0.5384615384615385 \\
\hline Subtypes_of_Asymmetric_Standard_Negation & 0.4277777777777778 \\
\hline Overlap_between_Situational_and_Epistemic_Modal_Marking & 0.44545454545454544 \\
\hline Comparative_Constructions & 0.6222222222222222 \\
\hline The_Future_Tense & 0.7 \\
\hline Perfective/Imperfective_Aspect & 0.5090909090909091 \\
\hline The_Perfect & 0.7 \\
\hline The_Past_Tense & 0.44545454545454544 \\
\hline Suppletion_in_Imperatives_and_Hortatives & 0.63 \\
\hline Exponence_of_Selected_Inflectional_Formatives & 0.63 \\
\hline Genitives,_Adjectives_and_Relative_Clauses & 0.5 \\
\hline Verbal_Number_and_Suppletion & 0.56 \\
\hline Suppletion_According_to_Tense_and_Aspect & 0.7 \\
\hline Reciprocal_Constructions & 0.5133333333333333 \\
\hline Inflectional_Synthesis_of_the_Verb & 0.7 \\
\hline Fusion_of_Selected_Inflectional_Formatives & 0.38888888888888895 \\
\hline Conjunctions_and_Universal_Quantifiers & 0.175 \\
\hline Exponence_of_Tense-Aspect-Mood_Inflection & 0.56 \\
\hline Obligatory_Double_Negation_in_SOV_languages & 0.233333333333333334 \\
\hline Obligatory_Double_Negation & 0.23333333333333334 \\
\hline Multiple_Negative_Constructions_in_SVO_Languages & 0.7 \\
\hline Double_negation_in_verb-initial_languages & 0.0 \\
\hline Internally-headed_relative_clauses & 0.7 \\
\hline Optional_Double_Negation_in_SVO_languages & 0.0 \\
\hline
\end{tabular}

